\title{
Functional Organization of the Temporal-Parietal Junction for Theory of Mind in Preverbal Infants: A Near-Infrared Spectroscopy Study
}

\author{
-Daniel C. Hyde, Charline E. Simon, Fransisca Ting, and Julia I. Nikolaeva \\ Department of Psychology, University of Illinois at Urbana-Champaign, Champaign, Illinois, 61820
}

Successful human social life requires imagining what others believe or think to understand and predict behavior. This ability, often referred to as theory of mind (ToM), reliably engages a specialized network of temporal and prefrontal brain regions in older children and adults, including selective recruitment of the temporal-parietal junction (TPJ). To date, how and when this specialized brain organization for ToM arises is unknown due to limitations in functional neuroimaging at younger ages. Here, we used the emerging technique of functional near-infrared spectroscopy to measure the functional brain response across parietal, temporal, and prefrontal regions in 7-month-old male and female infants as they viewed different video scenarios of a person searching for a hidden object. Over different conditions, we manipulated whether the person held an accurate (true) or inaccurate (false) belief about the location of the hidden object in the videos. In two separate experiments, we observed that activity from the TPJ, but not other temporal and prefrontal regions, spontaneously tracked with the beliefs of the other person, responding more during scenarios when the other person's belief regarding the location of the object was false compared with scenarios when her belief was true. These results mirror those obtained with adults to show that the TPJ already shows some functional organization relevant to high-level social cognition by around 7 months of age. Furthermore, these results suggest that infants may draw on similar core mechanisms to implicitly track beliefs, as adults do when reasoning explicitly about them.

Key words: infant; near-infrared spectroscopy; temporal lobe; temporal parietal junction; theory of mind

\section{Significance Statement}

Humans selectively engage a network of brain regions, including the temporal-parietal junction (TPJ), to track what others think, an ability referred to as theory of mind. How and when this specialized brain organization for high-level social cognition arises is unknown. Using the emerging technique of near-infrared spectroscopy with 7-month-old infants, we observed that activity of the TPJ, but not other temporal and frontal regions, distinguished between scenarios when another person's belief about the location of the object was false compared with scenarios when the belief was true. These results suggest that a basic neural architecture to understand and predict the actions of others based on their beliefs may be present from the first year of life.

\section{Introduction}

Humans consider the mental states, such as knowledge, desires, and goals, to interpret and predict the behavior of others. This

\footnotetext{
Received Jan. 27, 2017; revised March 9, 2018; accepted March 21, 2018.

Author contributions: D.C.H. wrote the first draft of the paper; D.C.H., C.E.S., J.I.N., and F.T. edited the paper; D.C.H., C.E.S., J.I.N., and F.T. designed research; D.C.H., C.E.S., J.I.N., and F.T. performed research; D.C.H., C.E.S., J.I.N., and F.T. analyzed data; D.C.H. wrote the paper.

We thank Danielle Branton, Yi Mou, and Lena Skalaban for their help with data collection and Renée Baillargeon and Rebecca Saxe for helpful comments on this manuscript.

The authors declare no competing financial interests.

Correspondence should be addressed to Daniel C. Hyde, Department of Psychology, University of Illinois

at Urbana-Champaign, 621 Psychology Building, 603 East Daniel Street, Champaign, IL 61820. E-mail: dchyde@illinois.edu.

DOI:10.1523/JNEUROSCI.0264-17.2018

Copyright $\odot 2018$ the authors $\quad 0270-6474 / 18 / 384264-11 \$ 15.00 / 0$
}

ability, often referred to as theory of mind (ToM), reliably engages a network of brain regions within the temporal-parietal junction (TPJ) and prefrontal cortex (PFC) (Frith and Frith, 2012; Gweon and Saxe, 2013; Koster-Hale and Saxe, 2013). Portions of the TPJ are of special interest in this network because they are recruited selectively for ToM in older children and adults (Deen et al., 2015). To date, however, little is known about how and when this specialized functional organization arises despite decades of behavioral research on and interest in ToM development (Gweon and Saxe, 2013).

At least two alternative predictions regarding functional organization for ToM can be drawn from the behavioral development literature. Around 3 to 5 years of age, children become able to explicitly answer questions regarding the inaccurate or false be- 
A True Belief (TB)

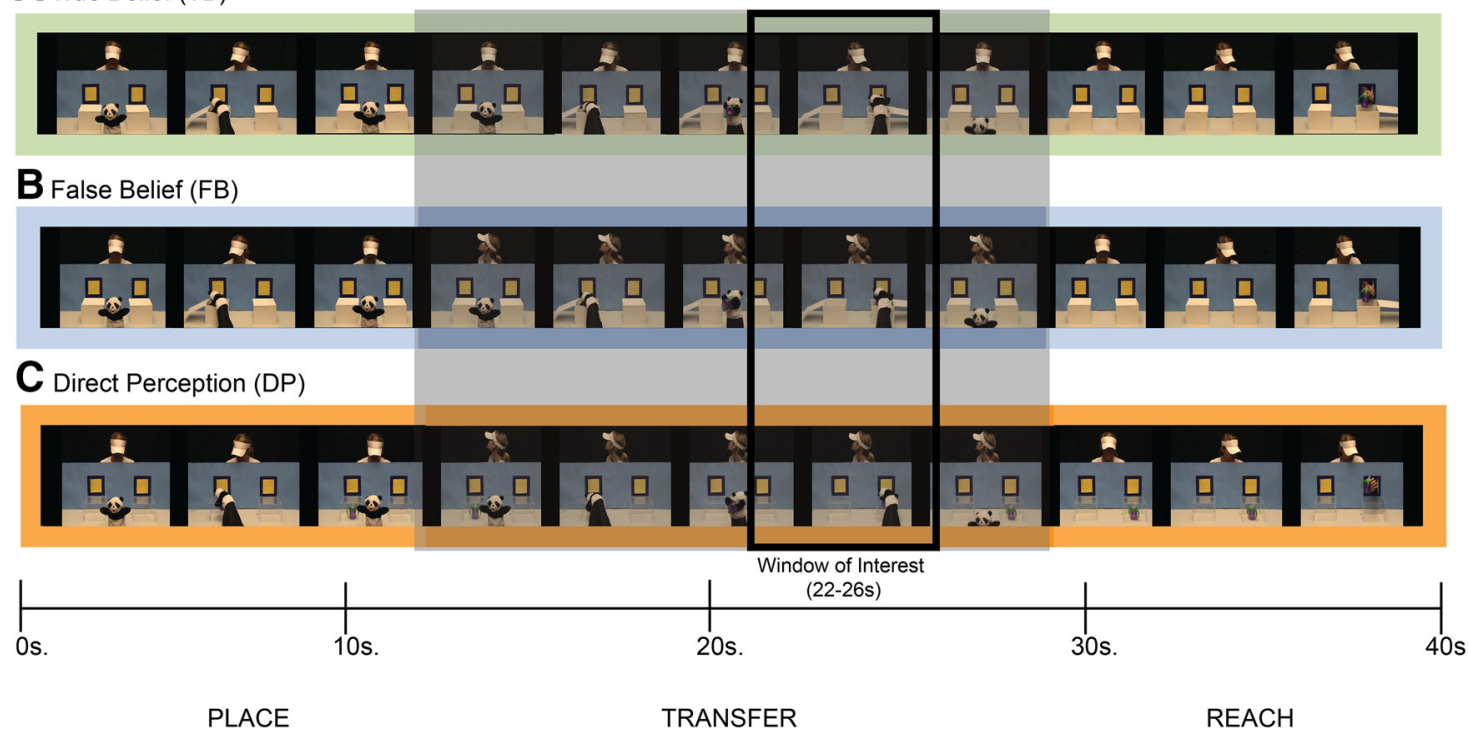

Figure 1. Overview of belief conditions presented through video clips. $\boldsymbol{A}$, In the TB condition, a person watches as a puppet hides an object in one of two boxes and then transfers it to the other box. After the transfer, the person reaches and successfully obtains the object. $\boldsymbol{B}$, In the FB condition, the person watches the puppet hide the object in the first box, but turns away before the puppet transfers the object to the other box. After the transfer, the person turns back toward the boxes and reaches successfully for the object. $\boldsymbol{C}$, In the DP condition, the person makes the same movements as in the FB condition, but the boxes are transparent, giving her access to the object's location. After the transfer, the person successfully reaches for the object. Below the depictions of the stimuli is an approximate timescale of events as they unfolded through the initial placement phase, the transfer phase, and the final reach phase. The gray box around the transfer phase highlights the window where the conditions differ conceptually. The smaller black outlined box represents the window of interest for analyzing the brain response derived from previous work with adults (Hyde et al., 2015).

liefs (FBs) of others (Wellman et al., 2001). Some interpret this as the major developmental milestone necessary for ToM to be considered present (Wellman et al., 2001; Apperly and Butterfill, 2009; Perner and Roessler, 2012). The prediction that follows from this view is that the specialized functional organization for ToM would require substantial brain development and should not be present until approximately the time children are able to explain their FB understanding. However, other work using implicit measures such as eye gaze suggest that even infants and toddlers may track the mental states of others, including FBs, well before they can explain them (Baillargeon et al., 2010). Some interpret these findings as evidence of early present ToM (Leslie et al., 2004; Baillargeon et al., 2010; Carruthers, 2013). What develops under this view is not ToM specifically, but rather the general cognitive abilities that support the increasing verbal and cognitive demands of explicit tasks. This alternative view predicts that specialized functional organization for ToM should be present very early in development and changes in behavioral competencies should be associated with maturation of brain mechanisms associated with domain general cognitive abilities. Although clear, these predictions have not been tested due to limitations in functional neuroimaging of high-level cognition in children younger than 3 years.

Here, we provide novel insight into early functional brain organization for ToM by applying functional near-infrared spectroscopy (fNIRS), a newly emerging methodological option (Gervain et al., 2011), to record from prefrontal and TPJ regions as 7-month-old infants watched implicit ToM video stimuli. Seven months was chosen because this is around the earliest age at which evidence for implicit belief tracking has been observed (Kovács et al., 2010; Southgate and Vernetti, 2014). Stimuli were video clips modeled after those used in behavioral studies with infants and the same as those used in a recent fNIRS study of ToM with adults (Southgate et al., 2007; Hyde et al., 2015). More spe- cifically, we showed a puppet hiding an object in one box and then transferring it to another box, after which a person attempted to retrieve it. Critically, across different clips, we manipulated the extent to which the person's belief about the location of the object was accurate (true) or false while controlling for lowerlevel stimulus features (see Materials and Methods). We reasoned that, if the brain is already organized for ToM within the first year of life, then activity in the TPJ should differentiate FB scenarios from accurate or true belief (TB) scenarios, a major diagnostic feature of ToM in behavior (Wellman et al., 2001) and what has been found using fNIRS with adults (Hyde et al., 2015). In contrast, if functional organization for ToM is not yet present around 7 months, then activity in the TPJ would not distinguish between scenarios involving true and FBs. In this case, implicit belief tracking might draw on different regions associated with other social (e.g., more anterior superior temporal regions) or general cognitive abilities (e.g., PFC) (Apperly and Butterfill, 2009).

\section{Materials and Methods}

\section{Experiment 1}

Participants. Participants were recruited from a database of local parents who had expressed interest in participating in our research. Twenty 7 -month-old infants made up the final dataset $\left(N=20, \mathrm{M}_{\text {age }}=7.37\right.$ months, $\mathrm{SD}_{\text {age }}=0.80$ months, 15 females). Five additional infants were excluded because they participated but contributed no useable data $(n=$ 4 , fussiness during probe placement or within first block; $n=1$, unable to obtain good signal due to hair). The study was conducted under the approval of the University of Illinois Office for the Protection of Human Subjects. Written informed consent was obtained from a parent or guardian of all children before data collection began. Families were given a small token of appreciation (book or t-shirt) or a travel reimbursement of 10 dollars for their participation.

Stimuli and design. After many previous studies of implicit ToM in infants and toddlers, stimuli were video clips of a person interacting with a puppet and an object in a goal-directed manner (Onishi and Baillar- 
geon, 2005; Southgate et al., 2007; Fig. 1). All runs started with an introductory event to familiarize the infant with the particular person, object, and puppet. Introductory events began with a novel person (always female) and a puppet waving, in turn, at the camera, after which the puppet picked up an object located at the center of a table and placed it in one of two boxes on the left and right sides of the table. The person then retrieved the object from the box to demonstrate a desire or goal to obtain it. After a black screen to reset, the object was shown again in the center of the screen between the two boxes with the person behind and the puppet in front. The puppet again picked up the object, placed it in the other box, and retreated from the stage, after which the person again retrieved it. After another brief black screen to reset, test events started.

Introductory events were followed by three test events. In all test events, the puppet picked up an object, put it in a box, and then moved the object to another box. All test events ended with the person successfully reaching for the object. Three distinct versions of test events, or conditions, were presented in each run, differing in the extent to which the person's belief was consistent with the object's actual location. In the TB condition (Fig. 1A), the person's belief about the object's location remained true throughout the video because she watched continuously as the puppet hid and then moved the object. In the FB condition (Fig. $1 B$ ), the person's belief about the location of the object became inconsistent with its actual location because she turned her head away shortly before the puppet transferred the object to the second box. In the direct perception (DP) control condition (Fig. 1C), the person also turned her head away as in the FB condition, but the boxes were clear, allowing perceptual access to the object's location. Even young infants have been shown to take into account whether a person has visual access to an object in interpreting and predicting their actions (Luo and Baillargeon, 2007; Luo and Johnson, 2009). Therefore, the DP condition was similar to the TB condition in that the person had some visual access to the object's location while matching the FB condition in lower-level body movements (e.g., head turn; for further logic regarding this control and for sample clips, see Hyde et al., 2015).

Each run contained an introductory event followed by each of the three test events (TB, FB, DP) presented in a random order. A total of four possible runs were presented involving a new person interacting with a new object each run.

Procedure. Infants sat on a parent or guardian's lap as they were fitted with flexible, custom-made headgear. Once the headgear, or probe, was placed, fitted, and adjusted for signal quality (details below), overhead lights were shut off and video clips were presented on a computer monitor approximately $90 \mathrm{~cm}$ away. We monitored looking behavior online for every infant participant using a closed circuit camera and paused between runs as needed. Stimuli were presented until the infant became inattentive, fussy, or after seeing four runs (each of three test conditions presented in each run).

fNIRS recordings. While video clips were being presented, we recorded the brain response using fNIRS. We used a TechEn CW6 (continuous wave) NIRS system with eight light (Four $690 \mathrm{~nm}$ and four $830 \mathrm{~nm}$ wavelength) sources and four light detectors to measure the cortical hemodynamic response at $50 \mathrm{~Hz}$ from the scalp. Sources and detectors were arranged into pairs with a fixed spacing of $2.5 \mathrm{~cm}$ to create a custom 12-channel probe covering scalp regions corresponding to inferior parietal, lateral temporal, and lateral PFC of the right hemisphere (six temporal-parietal channels and six frontal channels; Fig. 2). The probe was embedded into a custom-made spandex cap and optodes were held in place by tight-fitting rubber grommets. In addition, sports headbands and Velcro were applied to each infant as needed to steady the head probe against movement and ensure that optodes stayed firmly against the scalp. Ten-foot optical fibers carried light to and from the system and the headgear.

Probes were placed relative to common scalp landmarks that correspond to known 10-20 system points (Fig. 2). Average head size of infants in the final dataset was $43.92 \mathrm{~cm}(\mathrm{SD}=1.06 \mathrm{~cm})$. More specifically, the anterior-most detector of the temporal portion of the probe was always aligned directly above the middle of the ear, falling just behind the T4 10-20 point/T8 10-10 point; the posterior-most channel was positioned toward the $\mathrm{P} 4$ landmark. The inferior-most channel of the frontal portion of the probe fell between the $\mathrm{C} 4$ and $\mathrm{F} 4$ positions on the scalp, with the superior-most channel of the frontal probe between the $\mathrm{Cz}$ and Fz scalp positions (Fig. 2). Based on fNIRS-MRI scalp atlases, our probe covered scalp regions associated with the TPJ, including angular gyrus, posterior and middle superior temporal regions, and frontal lobe, including portions of superior frontal, dorsal-lateral prefrontal, and middle frontal cortical regions (Matsui et al., 2014; Lloyd-Fox et al., 2014). Signal strength and quality at each channel was monitored and adjusted as needed so that an attenuation value at the beginning of the recording was between 60 and $140 \mathrm{db}$ for at least $75 \%$ of the channels.

Data processing. Data processing was conducted using freely available Homer2 package (Huppert et al., 2009; version 2.1.0) in MATLAB version R2014a (MathWorks). We followed a similar approach to preprocessing as taken in our previously published fNIRS studies (Hyde et al., 2010, 2015; Edwards et al., 2016). To start, each run of the experiment was isolated from the continuous data and processed as a separate file to ensure that normalization of a given run was not skewed by more or less noisy portions of the data occurring at other times in the experiment. Raw intensity data from each channel in each run were subjected to an automatic pruning algorithm to identify and reject from further analysis any channel with too high of a signal (mean intensity $>1 \times 10^{7}$ ), too low of a signal (mean intensity $\leq 0$ ), insufficient signal-to-noise ratio (mean intensity/SD of intensity $<2$ ), or source detector separation of $<2$ or $>3$ $\mathrm{cm}$. Intensity data were then normalized and converted to optical density units. To correct probe-wide artifacts such as movement-induced signal changes, a principle components analysis was applied to identify objectively and filter changes in the signal common to all channels with the constraint to remove no more than $90 \%$ of the total variance of a given subject's data (Cooper et al., 2012). Resulting data were then low-pass filtered at $0.5 \mathrm{~Hz}$ to remove higher-frequency noise in the data. A final automatic algorithm was applied to detect and flag remaining portions of data containing evidence of movement artifact, where movement was defined as a change in the signal $>0.3$ optical density units over a given sample (0.2 s) (Strangman et al., 2003; Huppert et al., 2009; Scholkmann et al., 2010; Cooper et al., 2012). Such artifacts were masked $2 \mathrm{~s}$ before to $2 \mathrm{~s}$ after the data sample(s) containing the artifact and trials ( -2 before to 42 after stimulus onset) that contained such masks were rejected from further analysis. Incomplete runs at the end of the experiment, often due to fussiness or inattentiveness, were also dropped from the analysis. Finally, data were converted from optical density units to oxygenated ( $\mathrm{HbO})$, deoxygenated, and total $\mathrm{Hb}$ concentration changes using a modified Beer-Lambert law (Obrig et al., 2000; Strangman et al., 2002).

Participants who completed at least one run behaviorally without becoming upset and for whom any remaining data were retained after preprocessing were included in our analysis. Participants in Experiment 1 contributed an average of 7.1 individual trials $(S D=2.47)$ to the final dataset. There appeared to be no differences in the total number of trials contributed to the final dataset between experimental conditions in Experiment 1 ( $\mathrm{DP}=47, \mathrm{~TB}=47, \mathrm{FB}=48)$.

Data analysis. We focused on changes in $\mathrm{HbO}$ because it has been shown to be a more sensitive and reliable measure than deoxygenated or total $\mathrm{Hb}$ concentration change and has been used successfully in other fNIRS studies with infants (Strangman et al., 2002; Lloyd-Fox et al., 2010; Gervain et al., 2011; Aslin et al., 2015).

Our main analysis concerned whether functional brain activity (changes in $\mathrm{HbO}$ ) differentiated false from TB scenarios, a critical signature of implicit ToM. We first determined whether any channels within the temporal probe responded more to $\mathrm{FB}$ scenarios than TB or control DP scenarios between 22 and $26 \mathrm{~s}$, the same functional pattern, timing, and localization observed in adults with the same stimuli (Hyde et al., 2015). We used a linear mixed-effects (LME) analysis on individual trials to account for the unbalanced nature of the dataset (e.g., different numbers of trials per condition per subject), as well as individual differences in global signal strength. Our LME analysis was implemented in R (version 3.3.2) with the lme4 package (Bates et al., 2015; version 1.1.12). Following the guidelines of Barr et al. (2013), we used the maximal random effects structure justified by our design (Barr et al., 2013). This included a fixed effects factor of belief condition (three levels: DP, TB, and FB) and a random effects structure including random intercepts for each subject 
A

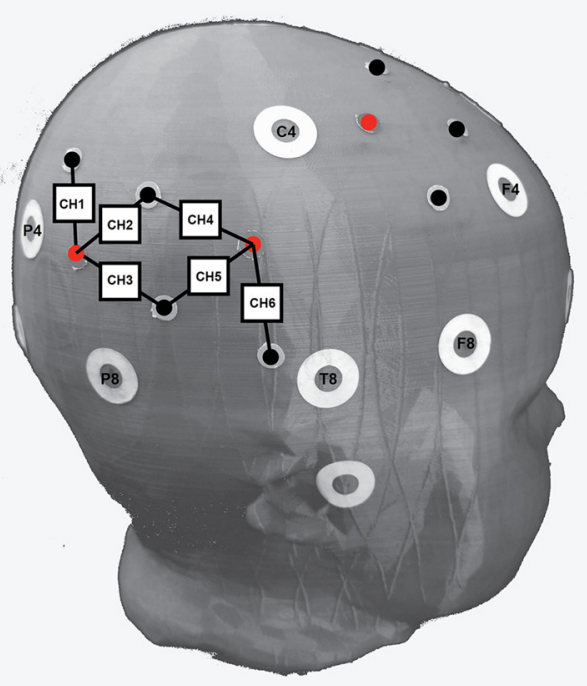

C

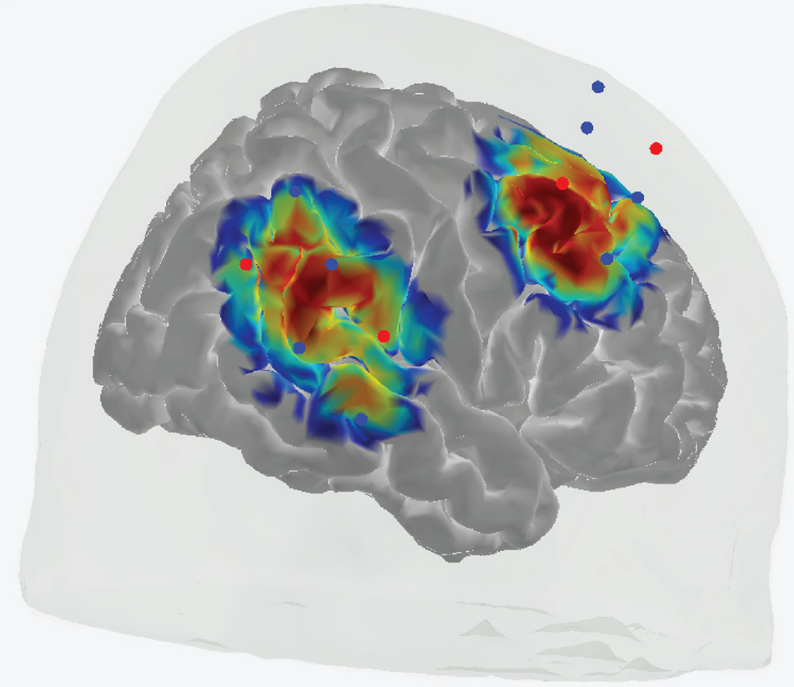

B

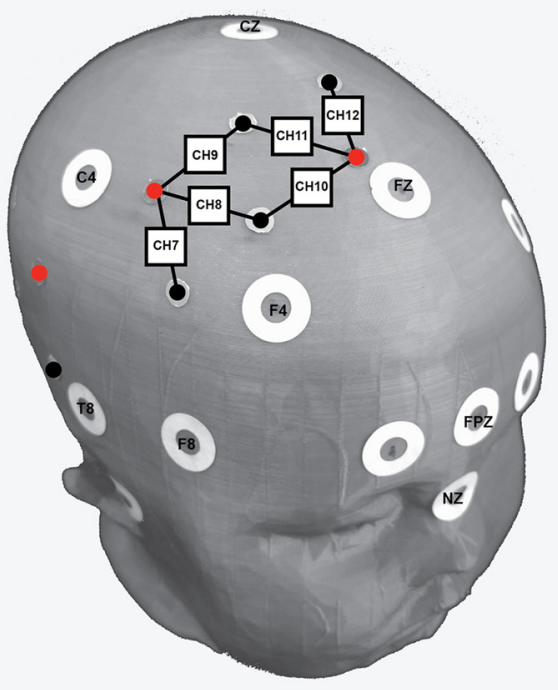

D

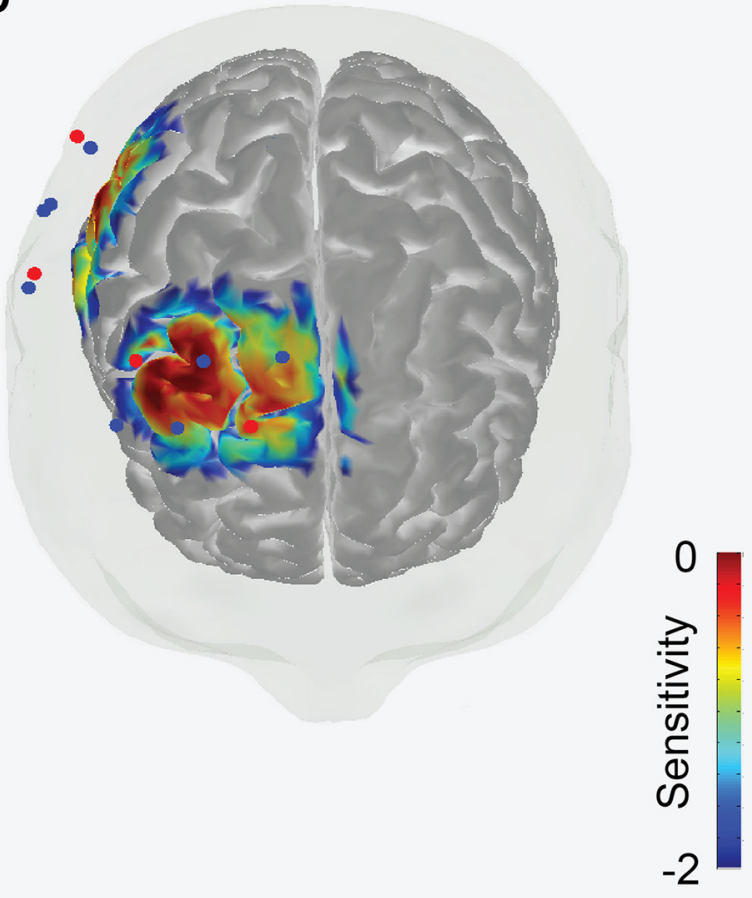

Figure 2. Experiment 1: fNIRS probe placement and sensitivity. $\boldsymbol{A}$, Depiction of temporal head probe positioning relative to common scalp landmarks. White circles represent $10-10$ scalp landmarks. Red circles represent light sources and black (or blue) circles represent light detectors. Black lines connecting light sources and detectors represent data channels. Square boxes with numbers are used to label each data channel 1-12. B, Depiction of frontal head probe positioning relative to common scalp landmarks. C, Sensitivity map $\left(\mathrm{mm}^{-1}\right)$ from lateral view derived from photon migration simulations of probe in a 3D head model (Materials and Methods for details). Values are displayed in $\log 10$ units. $\boldsymbol{D}$, Sensitivity map from the superior view.

and random slopes of condition by subject as follows: full model $=$ channel $\mathrm{HbO}$ data $\sim$ condition $+(1+$ condition|subject $)$. Item-level random effects were not justified by the highly unbalanced design and all attempts to model them resulted in failures in model convergence. In cases of nonconvergence of the full model, we used a backward selection procedure of reducing the complexity of the random effects structure by dropping the random slopes and rerunning the analyses (Barr et al., 2013). If this analysis yielded different results, we report statistics with the less complex random effects structure. If comparable results were obtained with the full random effects structure and the less complex random effects structure, statistics from the model with the full random effects structure are reported. To determine statistical significance, we first con- ducted a likelihood ratio test (LRT) comparing the experimental model with fixed and random effects to a reduced model that only included the random effects to obtain a test statistic $\left(\chi^{2}\right)$. We then conducted nonparametric permutation tests using LRT statistics derived from 5000 random permutations of data (randomly permuting condition labels within subjects) to calculate significance ( $p_{\mathrm{N}}$ values) at each channel. Because our infant head probe differed in optode spacing, optode arrangement, and scalp placement from that used in recent, similar study with adults (Hyde et al., 2015), a single TPJ channel of interest (COI) was not able to be determined a priori. Therefore, a Bonferroni-corrected $\alpha$ level was used as the threshold for statistical significance to correct for multiple channel comparisons $\left(p_{\mathrm{N}}=0.05 / 6\right.$ channels in temporal probe or 
Table 1. Results of analyses from all data channels in Experiment 2

\begin{tabular}{lllll}
\hline Channel & Region & MNI & $\chi^{2}$ & $p_{\mathrm{N}}$ \\
\hline 1 & Angular gyrus & $58-5043$ & 5.79 & 0.0228 \\
2 & Angular gyrus & $58-4731$ & 2.33 & 0.256 \\
3 & Superior temporal & $56-4924$ & 8.87 & $0.0038^{*}$ \\
4 & Superior temporal & $56-2924$ & 5.02 & 0.0558 \\
5 & Superior temporal & $46-3518$ & 5.47 & 0.042 \\
6 & Superior temporal & $51-2012$ & 7.06 & 0.0138 \\
7 & Middle frontal & $49 \quad 2041$ & 5.07 & 0.05 \\
8 & Middle frontal & 392146 & 4.02 & 0.0906 \\
9 & Middle frontal & $32 \quad 1447$ & 2.34 & 0.2528 \\
10 & Superior frontal & $29 \quad 3756$ & 0.01 & 0.9866 \\
11 & Superior frontal & $22 \quad 2857$ & 0.03 & 0.978 \\
12 & Superior frontal & $15 \quad 2963$ & 1.09 & 0.534 \\
\hline
\end{tabular}

MNI coordinates represent estimated locus of peak sensitivity. $\chi^{2}$ statistic results from likelihood ratio tests between full model and reduced model with only random effects. $p_{\mathrm{N}}$ are $p$-values from permutation tests $(\alpha=0.0083)$. It is important to note that the actual cortical sources for each channel are likely more medial in the infant brain compared with the estimates derived from an adult brain model here. * Indicates statistical significance.

$\left.p_{\mathrm{N}}=0.0083\right)$. Planned follow-up contrasts were made using the multcomp package (version 1.4-6) on estimated model coefficients from channels in which statistical significance in the overall fixed effect of mental state condition was found. Again, Bonferroni-corrected $\alpha$ levels were used to determine statistical significance in contrasts with correction for multiple condition comparisons (e.g., pairwise comparison between conditions $p=0.05 / 3$ or $p=0.0167$ ). The same analysis approach with the same significance thresholds was conducted independently for the frontal portion of the probe. The reported descriptive statistics were calculated by aggregating over trials for each condition for each participant (unless otherwise noted).

Additional exploratory analyses supplementary to our primary analysis followed to determine whether and/or to what extent other time windows might also show trending effects of belief sensitivity. For this exploratory analysis, we considered the average hemodynamic response before (2-22s) and after (26-42s) our main time window of interest at all channels. The same statistical significance thresholds were used for this analysis as in the main analysis. However, any resulting effects were only meant to provide some characterization of data in other channels and time windows, so any potential trends were to be treated with caution.

Estimating cortical sensitivity of data channels. In addition to using infant scalp fNIRS-MRI atlases to guide probe placement and thereby estimate from what brain region each channel was measuring (Matsui et al., 2014; Lloyd-Fox et al., 2014), we also produced a sensitivity map by combining spatial registration of our own probe with the structural atlas and forward modeling of photon migration (tMCimg) (Boas et al., 2002) implemented in AtlasViewer version 2.1 (Custo et al., 2010; Aasted et al., 2015). More specifically, we estimated the cortical sensitivity profile of each channel in our probe by simulating photon migration in a realistic, mesh-based 3D head model (1 million photons for each channel, see Fang, 2010 for algorithmic details). Montreal Neurological Institute (MNI) coordinate estimates for the center of sensitivity for each channel were obtained from the forward models (Table 1).

\section{Experiment 2}

In a second experiment, we attempted to replicate and extend the results of Experiment 1 using a preregistered research plan (https://osf.io/ t9gvr/). The experimental design, procedures for data quality checking and data recording, data processing, data reduction and elimination, and the fixed array data analysis were prespecified and almost entirely the same as those in Experiment 1. The main difference was that we used a modified optical head probe (Fig. 3). More specifically, we measured over the same regions of the right TPJ, as well as a new prefrontal region, the left medial PFC (mPFC), as a new group of infants of the same age watched the same stimulus events presented in Experiment 1. Our purpose was twofold: (1) to assess the reproducibility of the TPJ sensitivity to beliefs observed in Experiment 1 and (2) to assess the sensitivity of a new region, left $\mathrm{mPFC}$, to beliefs in 7-month-old infants. Because a majority of methodological details were the same as those in Experiment 1, we primarily note differences below.

Participants. We planned and collected useable data on a larger sample of infants $\left(N=30 ; \mathrm{M}_{\text {age }}=7.91\right.$ months, $\mathrm{SD}_{\text {age }}=0.76$ months, 10 females). An additional 11 infants were tested but did not produce useable data ( $n=9$, fussiness during probe placement or within first block; $n=2$, incorrect head probe placement).

Video monitoring of behavior. In addition to online monitoring for visual attention to stimuli to determine when to stop the experiment as in Experiment 1, we also recorded video of looking behavior for post hoc consideration. We obtained full video recordings of all trials for 28/30 infants, with partial recordings $(n=1)$ or no recordings $(n=1)$ because of technical difficulties for 2/30 infants. Available video recordings from testing sessions were examined frame by frame and coded by two trained, independent coders. To determine the proportion of looking, we divided the number of frames in which both coders agreed that participants were looking at the video stimuli by the total number of frames. Interrater reliability of coding (looking/not looking) was very high ( $\mathrm{M}=98 \%$, $\mathrm{SD}=4 \%)$. On average, infants looked $84 \%$ (SD = 15\%) of the time test videos were playing. Such high levels of attention likely resulted from our procedure of stopping the testing session when infants became bored, inattentive, or upset. There appeared to be no difference in the percentage of looking to test videos between belief conditions $(\mathrm{DP}=85 \%$; $\mathrm{TB}=$ $83 \% ; \mathrm{FB}=84 \%)$. On only $3 \%(5 / 162)$ of trials did infants look less $50 \%$ of the time.

fNIRS recordings. Probes were first placed in reference to 10-20 scalp landmarks over right temporal regions in the same manner as in Experiment 1 (above Materials and Methods and Fig. 2) and then placed over scalp landmarks corresponding to left frontal regions (Fig. 3). Source detector spacing remained at $2.5 \mathrm{~cm}$ for the temporal portion of the probe as in Experiment 1, but was extended to $3 \mathrm{~cm}$ for the frontal portion of the probe. Frontal placement involved centering the two source optodes on either side of the Fp1 10-20 scalp position, thereby positioning detector optodes between the midline frontal scalp sites and the lateral F3 fontal scalp site (Fig. 3A). Cortical sensitivity of frontal channels was estimated in the same way as all channels in Experiment 1 (Fig. 3B, Table 2). The average head size of infants in the final dataset of Experiment 2 was $44.43 \mathrm{~cm}$ $(\mathrm{SD}=1.34 \mathrm{~cm})$.

Data processing. Data processing and rejection parameters were prespecified beforehand and were the same as in Experiment 1. Processing and rejection was conducted separately for the temporal and frontal portions of the probe given that the source detector spacing was different between them. After processing and automatic rejection of artifact-laden data, participants contributed an average of 5.73 individual trials $(\mathrm{SD}=$ $2.46)$ to the final temporal lobe dataset and $5.76(\mathrm{SD}=2.45)$ for the final frontal lobe dataset. There appeared to be no differences in the total number of trials contributed to the final dataset between the experimental conditions of Experiment 2 (temporal lobe: $\mathrm{DP}=58, \mathrm{~TB}=57, \mathrm{FB}=$ 57; frontal lobe: $\mathrm{DP}=58, \mathrm{~TB}=57, \mathrm{FB}=58$ ).

Looking behavior from video recordings was not used for data rejection in the primary analyses because this was not in the prespecified plan. Furthermore, exploratory analyses eliminating trials when infants did not look over $50 \%$ of the trial, which only made of $3 \%$ of data, did not change any of the results reported below. Therefore, we forego any further fNIRS data exclusion based on video recordings.

Data analyses. The primary analysis of Experiment 2 followed a preregistered analysis plan closely matching the approach of Experiment 1 to test for left mPFC sensitivity to beliefs and exactly matching the approach of Experiment 1 in an attempt the reliability of the findings of right TPJ sensitivity to beliefs in infants (for details, see: https://osf.io/t9gvr/).

We also preregistered two other types of exploratory analyses to test sensitivity of the new portion of our probe covering left mPFC. First, we planned to explore sensitivity to our belief conditions in eight $4 \mathrm{~s}$ time windows before and after the a priori time window of interest (22-26 s) with a similar statistical approach as that used in our main time window of interest analysis. Second, we planned to analyze whether the average peak $\mathrm{HbO}$ response increased above baseline for any of the mPFC channels. To do this, we found the peak of the $\mathrm{HbO}$ response between 2 and $42 \mathrm{~s}$ for each trial for each subject, extracted the average response $\pm 2 \mathrm{~s}$ 
A

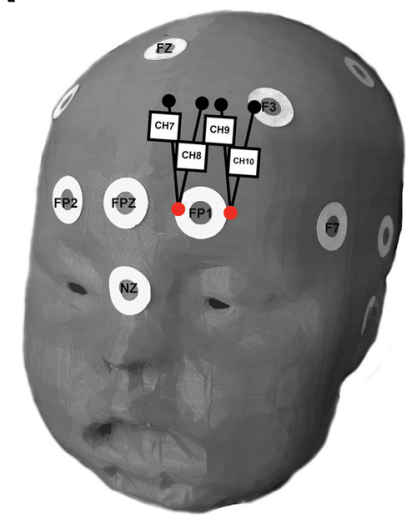

B

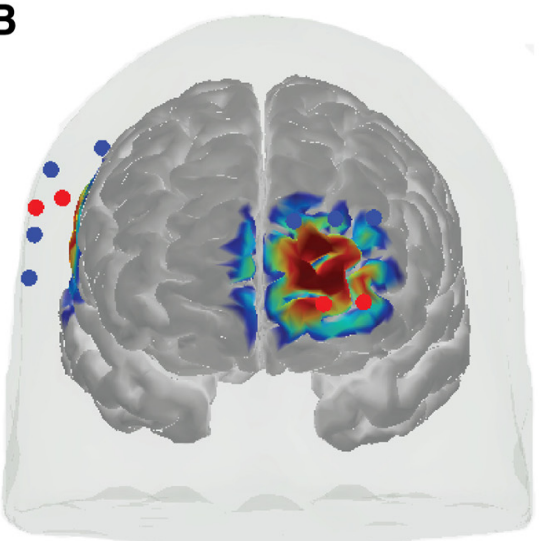

condition compared to the TB condition $(\beta=-0.64, \mathrm{SE}=0.26, z=-2.46, p=$ $0.014)$ and the DP control condition $(\beta=$ $-0.83, \mathrm{SE}=0.29, z=-2.86, p=0.0042$; DP vs TB: $\beta=0.19, \mathrm{SE}=0.30, z=0.63$, $p=0.527$; Fig. $4 A$ ). A closer look at the full time course of activity within this channel revealed that, whereas activity increased for all conditions during the earlier phases of the test trial, only activity in the FB condition remained elevated at the beginning of the second half of the test trial $\left(\mathrm{M}_{\mathrm{FB}}=0.948\right.$ $\mu \mathrm{M}, \mathrm{SD}_{\mathrm{FB}}=1.281 \mu \mathrm{M} ; \mathrm{M}_{\mathrm{TB}}=0.0282 \mu \mathrm{M}$, $\mathrm{SD}_{\mathrm{TB}}=0.8603 \mu \mathrm{M} ; \mathrm{M}_{\mathrm{DP}}=0.0698 \mu \mathrm{M}$, $\mathrm{SD}_{\mathrm{DP}}=1.118 \mu \mathrm{M}$; Fig. $4 B$ ). Estimates of probe sensitivity from photon migration simulations and comparison of channel scalp locations to established infant scalp surface maps using fNIRS-fMRI (LloydFox et al., 2014) suggest that activity in this channel likely arises from a posterior superior temporal region within the TPJ

Figure 3. Experiment 2 fNIRS frontal probe placement and sensitivity. $A$, Depiction of frontal head probe positioning relative to common scalp landmarks. White circles represent 10-10 scalp landmarks. Red circles represent light sources and black (or blue) circles represent light detectors. Black lines connecting light sources and detectors represent data channels. Square boxes with numbers are used to label each data channel 7-10. , Sensitivity map $\left(\mathrm{mm}^{-1}\right)$ from frontal view derived from photon migration simulations of probe in a 3D head model (Materials and Methods for details). Values are displayed in $\log 10$ units.

Table 2. Results of primary analyses of mPFC channels in Experiment 2

\begin{tabular}{lllll}
\hline Channel & Region & MNI & $\chi^{2}$ & $p_{\mathrm{N}}$ \\
\hline 7 & Superior medial frontal & -11638 & 0.92 & 0.554 \\
8 & Superior frontal & -16647 & 2.49 & 0.197 \\
9 & Superior medial frontal & -14524 & 1.39 & 0.454 \\
10 & Middle frontal & -25657 & 3.21 & 0.142 \\
\hline
\end{tabular}

MNI coordinates represent estimated locus of peak sensitivity. $\chi^{2}$ statistic results from likelihood ratio tests between full model and reduced model with only random effects during the a priori time window of interest. $p_{\mathrm{N}}$ are $p$-values from permutation tests $(\alpha=0.0125)$. It is important to note that the actual cortical sources for each channel are likely more medial in the infant brain compared with the estimates derived from a mature brain model here.

surrounding the peak, averaged the responses across the entire dataset, and tested that value against zero (baseline) using a $t$ test.

\section{Additional analyses}

Additional exploratory analyses were conducted to better understand our results after they were obtained. These included a reanalysis of the data using a functionally defined COI (fCOI) approach (Powell et al., 2017a) to supplement the primary, fixed array analysis used in both experiments. To do this, we defined a fCOI for each run for each participant as the channel showing the greatest response to the $\mathrm{FB}$ condition compared with the average of the TB and DP conditions $[\mathrm{FB}-(\mathrm{TB}+$ $\mathrm{DP} / 2)]$ across the average of all other independent runs from that participant. The search was anatomically restricted to the three most posterior channels of the temporal probe (channels 1-3), given their estimated sensitivity and our primary focus of identifying functional response differences from the TPJ and not more anterior temporal regions. This search was done iteratively for each run for each participant so that the fCOI for each run was defined from independent data from that particular participant. In cases in which only one run of useable data was obtained, we used the channel showing differential sensitivity to beliefs from the primary fixed array analysis of Experiment 1 (channel 3; see Results section) as the fCOI. Once an fCOI was defined from independent data for a given run, data were extracted for each condition from that run and analyzed using the same LME analysis approach as the primary analysis.

\section{Results}

\section{Experiment 1}

An analysis of the hemodynamic response in temporal channels during the a priori time window of interest (22-26 s) revealed robust sensitivity to the belief conditions in a single TPJ channel (channel 3, $\chi^{2}(2)=8.87, p_{N}=0.0038$; Fig. 4). Model contrasts in this channel showed that the $\mathrm{HbO}$ response was larger to the $\mathrm{FB}$
(Fig. 4C). The estimated locus of maximal channel sensitivity (MNI: 56, -49, 24; also see Table 1) is consistent with localization of peak fMRI activity found to engage selectively for ToM in adults (Deen et al., 2015). No additional differential sensitivity between belief conditions was observed in any other temporal or frontal channels after strict correction for multiple comparisons (all $p_{\mathrm{N}}>0.01$; Table 1). Additional exploratory analyses also failed to find any large differences between belief conditions in temporal or frontal regions during earlier (2-22s) or later (26-42s) time windows (all $p_{\mathrm{N}}>0.03$ ). Therefore, differences between belief conditions appeared to be largely restricted to a single TPJ channel and time window directly surrounding the transfer portion of video clips when the person's belief became inconsistent with the actual object location, as was observed in a previous study with adults (Hyde et al., 2015).

\section{Experiment 2}

In Experiment 2, we attempted to extend measurements to another region of the brain thought to be important to ToM, the mPFC, as well as to test the reliability of the TPJ findings of Experiment 1.

\section{Medial prefrontal sensitivity to beliefs}

An analysis of the hemodynamic response over left mPFC regions using a preregistered analysis plan revealed no global sensitivity to the different belief conditions in any of the four channels during the a priori time window of interest $\left(22-26 \mathrm{~s}\right.$, all $p_{\mathrm{N}}>0.14$; see Table 2 for full statistics). Further exploratory analysis of the peak $\mathrm{HbO}$ response of each channel revealed that only the most lateral frontal channel in the probe (channel 10) had a peak HbO response greater than baseline after correcting for multiple channel analyses $\left(\alpha\right.$ level $=0.0125$; channel 10: $\left.t_{(57)}=3.46, p=0.001\right)$. However, the average timing of the peak response was much earlier (2.69 s) than would be expected if it were a response to the test stimulus given the known delay in the hemodynamic response (channel 7: $t_{(57)}=1.53, p=0.132$; channel 8: $t_{(57)}=2.00$, $p=0.050$; channel 9: $\left.t_{(57)}=2.31, p=0.024\right)$. A final set of exploratory analyses of $\mathrm{mPFC}$ data in other time windows, uncorrected for multiple comparisons, revealed differential sensitivity to the belief conditions only in the most lateral mPFC channel (10) during the time window between 18 and 

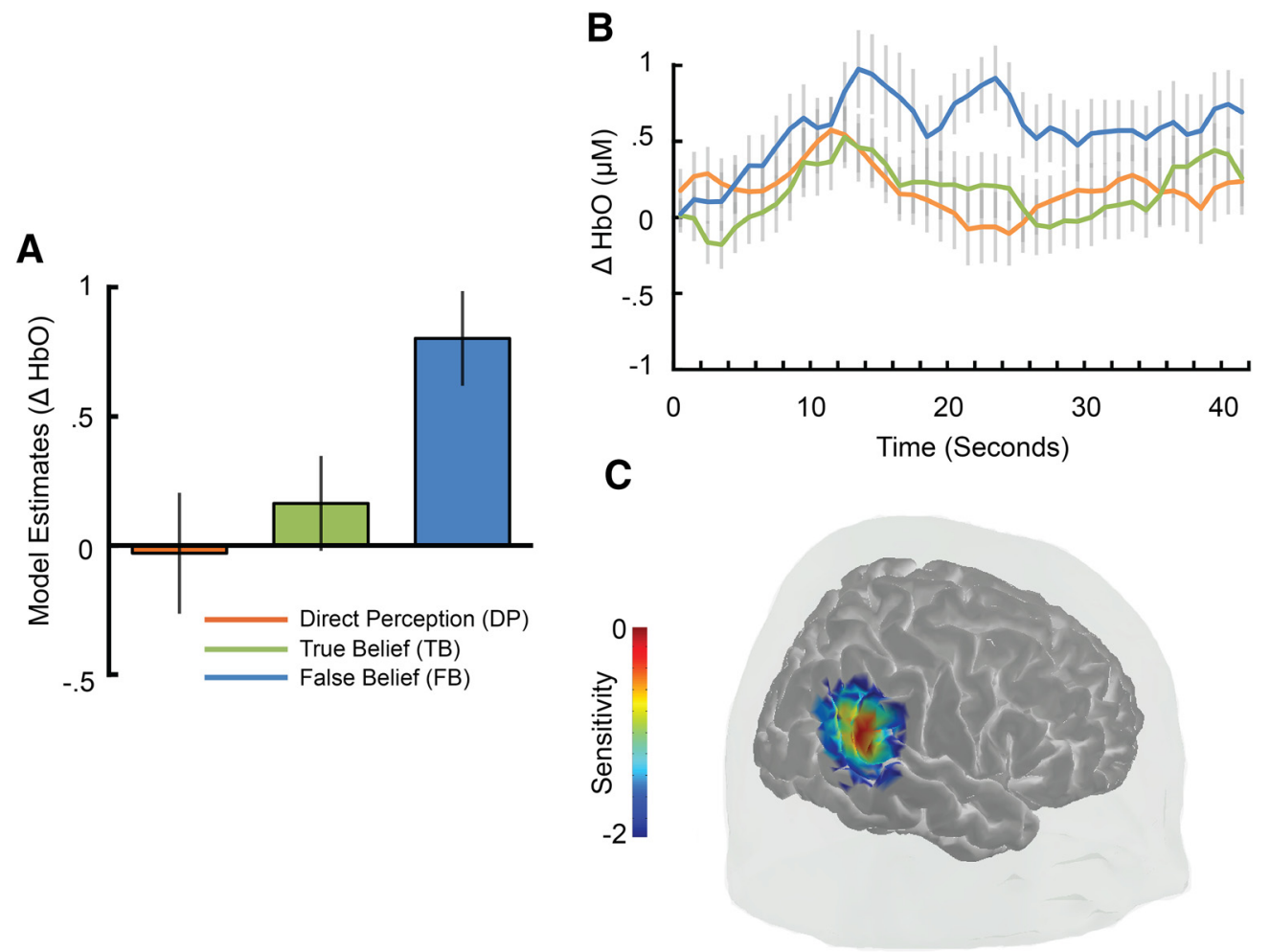

Figure 4. Experiment 1 results showing functional sensitivity to the beliefs of others in the TPJ. $A$, Estimates of least-squares means from the linear mixed model of belief condition on neural response of channel 3 between 22 and 26 s. B. Time course of change in activity in channel 3 over the entire duration of the test videos. C, Cortical sensitivity estimates ( $\mathrm{mm}^{-1}$ ) for channel 3 displayed in $\log 10$ units (see Materials and Methods for additional details). Error bars indicate \pm 1 SE.

$22 \mathrm{~s}\left(\chi^{2}(2)=7.30, p_{\mathrm{N}}=0.012\right)$. Pairwise model contrasts showed that the $\mathrm{HbO}$ response to the TB condition was greater than both the FB condition and the DP condition (FB vs TB: $\beta=0.32, \mathrm{SE}=$ $0.14, z=2.20, p=0.028$; DP vs TB: $\beta=0.42, \mathrm{SE}=0.15, z=2.78$, $p=0.005)$. The timing of this effect was slightly earlier than that seen in temporal lobe and the pattern of response suggests that this region of the $\mathrm{mPFC}$ may respond more to direct or joint attentional gaze present in the TB condition $\left(\mathrm{M}_{\mathrm{TB}}=0.1815 \mu \mathrm{M}\right.$, $\left.\mathrm{SD}_{\mathrm{TB}}=0.7173 \mu \mathrm{M}\right)$ compared with the averted gaze in the $\mathrm{FB}$ and $\mathrm{DP}$ conditions $\left(\mathrm{M}_{\mathrm{FB}}=-0.1291 \mu \mathrm{M}, \mathrm{SD}_{\mathrm{FB}}=0.6184 \mu \mathrm{M} ; \mathrm{M}_{\mathrm{DP}}=\right.$ $\left.-0.2229 \mu \mathrm{M}, \mathrm{SD}_{\mathrm{DP}}=0.6630 \mu \mathrm{M}\right)$. However, given the exploratory nature of this analysis, including the large number of statistical test runs and the lack of statistical correction for them, these results should be treated with much caution. No other indication of differential sensitivity between conditions was seen in any other $\mathrm{mPFC}$ channels and/or time windows (all $p_{\mathrm{N}}>0.06$; see Table 3 for full results).

\section{Temporal sensitivity to beliefs}

An analysis of the hemodynamic response in our a priori TPJ COI (channel 3) during the a priori time window of interest (22-26 s) using the preregistered analysis plan replicated the differential sensitivity to the belief conditions (channel 3, $\chi^{2}(2)=6.20, p_{\mathrm{N}}=$ 0.021; Fig. 5) seen in Experiment 1. Model contrasts showed that the $\mathrm{HbO}$ response in this channel was larger for the $\mathrm{FB}$ condition compared with the TB condition $(\beta=-0.42$, $\mathrm{SE}=0.16, z=$ $-2.60, p=0.009 ; \mathrm{M}_{\mathrm{FB}}=0.2351 \mu \mathrm{M}, \mathrm{SD}_{\mathrm{FB}}=.7216 \mu \mathrm{M} ; \mathrm{M}_{\mathrm{TB}}=$ $\left.-0.1484 \mu \mathrm{M}, \mathrm{SD}_{\mathrm{TB}}=.7751 \mu \mathrm{M}\right)$ and that there were no differences in the $\mathrm{HbO}$ response between the TB and DP condition, exactly as in Experiment 1 (DP vs TB: $\beta=-0.28, \mathrm{SE}=.16, z=$ $\left.-1.77, p=0.076 ; \mathrm{M}_{\mathrm{DP}}=0.0896 \mu \mathrm{M}, \mathrm{SD}_{\mathrm{DP}}=.7365 \mu \mathrm{M}\right)$. We did not, however, observe a statistically significant difference be- tween the FB and DP conditions $(\beta=-0.15, \mathrm{SE}=0.16, z=$ $-0.92, p=0.359$; Fig. 5), as we did in Experiment 1 .

Prespecified exploratory analyses also revealed an effect of belief condition in one other channel adjacent to the COI (channel 2, $\left.\chi^{2}(2)=7.61, p_{N}=0.0096\right)$. Model contrasts showed that the response to the $\mathrm{FB}$ condition was larger than that to the $\mathrm{TB}$ condition $\left(\beta=-0.44, \mathrm{SE}=0.16, z=-2.80, p=0.005 ; \mathrm{M}_{\mathrm{FB}}=\right.$ $0.1676 \mu \mathrm{M}, \mathrm{SD}_{\mathrm{FB}}=0.5790 \mu \mathrm{M} ; \mathrm{M}_{\mathrm{TB}}=-0.3769 \mu \mathrm{M}, \mathrm{SD}_{\mathrm{TB}}=$ $0.6828 \mu \mathrm{M})$, but no different from the DP condition $(\beta=-0.23$, SE $0.16, z=-1.45, p=0.15$; DP vs TB: $\beta=-0.21$, SE $=0.16$, $\left.z=-1.31, p=0.19 ; \mathrm{M}_{\mathrm{DP}}=-0.0958 \mu \mathrm{M}, \mathrm{SD}_{\mathrm{DP}}=.8709 \mu \mathrm{M}\right)$. This pattern mirrored that observed in the a priori COI.

\section{Additional analyses}

While a majority of the temporal probe findings of Experiment 1 directly replicated in Experiment 2, two main differences were observed. Experiment 1 showed that the infant TPJ differentiated the FB condition from both the TB and DP condition in a single channel, whereas Experiment 2 only showed TPJ differentiation between FB and TB in two adjacent channels. Therefore, we followed up with several additional post hoc analyses to better understand this difference.

Functional COI analysis of temporal sensitivity to beliefs

Based on our experience placing the probe in both experiments and the fact that sensitivity was found to be more widespread in Experiment 2, we suspected that more variation in probe placement could have led to less clear results in Experiment 2. Specifically, we found consistent scalp placement to be more difficult when spanning both hemispheres with the bilateral head probe of Experiment 2 compared with the fully right lateralized head probe used in Experiment 1. To test this idea, we applied an fCOI 
Table 3. Results of exploratory analyses of mPFC channels in Experiment 2

\begin{tabular}{lllllllll}
\hline & \multicolumn{7}{l}{ Time window $(\mathrm{s})$} \\
\cline { 2 - 9 } Channel & $6-10$ & $10-14$ & $14-18$ & $18-22$ & $26-30$ & $30-34$ & $34-38$ & $38-42$ \\
\hline 7 & 0.902 & 0.684 & 0.673 & 0.099 & 0.218 & 0.703 & 0.282 & 0.928 \\
8 & 0.881 & 0.875 & 0.498 & 0.123 & 0.323 & 0.935 & 0.706 & 0.802 \\
9 & 0.739 & 0.497 & 0.600 & 0.279 & 0.370 & 0.779 & 0.950 & 0.960 \\
10 & 0.352 & 0.598 & 0.253 & 0.012 & 0.065 & 0.526 & 0.393 & 0.327 \\
\hline
\end{tabular}

Reported statistics for each channel during each time window are $p_{N}$ values from permutation tests of the likelihood ratio tests between the full model and the reduced model with only random effects.

analysis method, which was shown recently to be more robust to subtle variations in infant fNIRS probe placements than fixed array analyses (Powell et al., 2017a), to data from both of our experiments. We reasoned that if differences in probe placement account for the differences in results between experiments, then applying the fCOI method to Experiment 2 data would result in increased sensitivity (compared with the fixed array analysis of Experiment 2) and subsequently replicate the full functional response pattern of belief sensitivity seen with the fixed array analysis of Experiment 1. As a further point of comparison, if probe placement was more consistent in Experiment 1, then reanalyzing it using the fCOI method should not change the pattern of results observed with the fixed array analysis.

As suspected, a reanalysis of Experiment 2 data using a TPJ fCOI approach revealed the full pattern of differential sensitivity to the belief conditions seen in fixed array analysis of Experiment 1, with an $\mathrm{HbO}$ response that was greater for the $\mathrm{FB}$ condition $\left(\mathrm{M}_{\mathrm{FB}}=0.3340 \mu \mathrm{M}, \mathrm{SD}_{\mathrm{FB}}=0.5661 \mu \mathrm{M}\right)$ than both the TB condition $\left(\mathrm{M}_{\mathrm{TB}}=-0.0542 \mu \mathrm{M}, \mathrm{SD}_{\mathrm{TB}}=0.8882 \mu \mathrm{M}\right)$ and $\mathrm{DP}$ condition $\left(\mathrm{M}_{\mathrm{DP}}=-0.0052 \mu \mathrm{M}, \mathrm{SD}_{\mathrm{DP}}=0.7892 \mu \mathrm{M}\right.$; Overall model: $\chi^{2}(2)=6.81, p_{\mathrm{N}}=0.036$; FB vs TB: $\beta=-0.39, \mathrm{SE}=0.16, z=$ $-2.37, p=0.018$; FB vs DP: $\beta=-0.36$, $\mathrm{SE}=0.16, z=-2.20$, $p=0.028$; DP vs TB: $\beta=-0.03$, SE $=0.16, z=0.19, p=0.853$; Fig. 6). A reanalysis of Experiment 1 data using a TPJ fCOI rather than the original fixed array analysis did not change the pattern of results. With the fCOI analysis of Experiment 1 data, we observed the full pattern of differential sensitivity to the belief conditions seen in the fixed array analysis of the same data $\left(\mathrm{M}_{\mathrm{FB}}=0.7978\right.$ $\mu \mathrm{M}, \mathrm{SD}_{\mathrm{FB}}=1.145 \mu \mathrm{M} ; \mathrm{M}_{\mathrm{TB}}=-0.0941 \mu \mathrm{M}, \mathrm{SD}_{\mathrm{TB}}=1.014 \mu \mathrm{M}$; $\mathrm{M}_{\mathrm{DP}}=0.0918 \mu \mathrm{M}, \mathrm{SD}_{\mathrm{DP}}=1.113 \mu \mathrm{M}$; Overall Model: $\chi^{2}(2)=$ 9.58, $p_{\mathrm{N}}=0.008$; FB vs TB: $\beta=-0.74, \mathrm{SE}=0.27, z=-2.75, p=$ 0.006 ; FB vs DP: $\beta=-0.73, \mathrm{SE}=0.27, z=-2.72, p=0.007$; DP vs TB: $\beta=-0.006, \mathrm{SE}=0.27, z=0.02, p=0.982)$. These data support the idea that more variation in probe placement in Experiment 2 contributed additional measurement noise and applying a more sensitive analysis method that could better account for this type variation resulted in a replication of the full pattern of sensitivity seen in Experiment 1 and in a previous study with adults (Hyde et al., 2015).

\section{Overall analysis of data combined from both experiments}

Finally, given that both samples were of limited size (Experiment 1, $n=20$; Experiment 2, $n=30$ ), we pooled temporal probe data from both experiments to test statistically whether the full pattern of functional sensitivity was robust across our entire study sample $(N=50)$ or if it was more contextual (i.e., interacted with experiment). A fixed array analysis on the combined dataset revealed a robust effect of belief condition on the hemodynamic response $\left(\chi^{2}(2)=13.11, p_{\mathrm{N}}=0.0002\right)$. Model contrasts showed that the $\mathrm{HbO}$ response was larger for the $\mathrm{FB}$ condition compared with both the $\mathrm{TB}$ condition $(\beta=-0.52, \mathrm{SE}=0.15, z=-3.48, p=$ $0.0005 ; \mathrm{M}_{\mathrm{FB}}=0.5204 \mu \mathrm{M}, \mathrm{SD}_{\mathrm{FB}}=1.034 \mu \mathrm{M} ; \mathrm{M}_{\mathrm{TB}}=-0.0763$ $\left.\mu \mathrm{M}, \mathrm{SD}_{\mathrm{TB}}=0.8069 \mu \mathrm{M}\right)$ and the DP control condition $(\beta=$
$-0.46, \mathrm{SE}=0.16, z=-2.86, p=0.004 ; \mathrm{DP}$ vs TB: $\beta=-0.06$, $\mathrm{SE}=0.16, z=0.38, p=0.701 ; \mathrm{M}_{\mathrm{DP}}=0.0817 \mu \mathrm{M}, \mathrm{SD}_{\mathrm{DP}}=0.8977$ $\mu \mathrm{M})$. There was also an effect of experiment, with the general $\mathrm{HbO}$ response being larger for Experiment 1 compared with Experiment 2 $\left(\beta=-0.32, \mathrm{SE}=0.13, \chi^{2}(2)=4.94, p_{\mathrm{N}}=0.029 ; \mathrm{M}_{\mathrm{E} 1}=0.3026\right.$, $\left.\mathrm{SD}_{\mathrm{E} 1}=1.344 ; \mathrm{M}_{\mathrm{E} 2}=0.0439 ; \mathrm{SD}_{\mathrm{E} 2}=0.9060\right)$. Crucially, however, there was no interaction between belief condition and experiment $\left(\chi^{2}(2)=5.11, p_{N}=0.089\right)$. Despite Experiment 2 contributing more participants, the functional response pattern seen with a fixed channel analysis of the combined dataset more closely matched the full functional pattern seen in Experiment 1 and that seen in a previous study with adults (Hyde et al., 2015) than that of the fixed channel analysis of Experiment 2. The results of this analysis provide further empirical support for the idea that the full pattern of sensitivity, with TPJ activity differentiating FB from both TB and DP, better characterizes the entire dataset than the more limited sensitivity seen in the fixed array analysis of Experiment 2.

\section{Discussion}

Over two experiments, an initial experiment and a replication experiment, we observed that the functional response of the TPJ in 7-month-old infants reliably and robustly distinguished scenarios involving a person with a FB from those involving a person with true or accurate beliefs with regard to the location of an object. These results provide the first empirical evidence to date that infants engage temporal-parietal brain mechanisms when viewing events claimed to evoke spontaneous (or implicit) ToM (Southgate et al., 2007). Furthermore, this particular pattern of neural sensitivity, distinguishing scenarios involving FBs from those involving TBs, mirrors a critical behavioral signature many agree is indicative of ToM (Wellman et al., 2001; Apperly and Butterfill, 2009). The timing and pattern of these differences were very similar to those observed in adults with the same stimuli and brain measure (Hyde et al., 2015). Furthermore, the localization of this temporal response was consistent with that observed in adults using fNIRS as well as previous fMRI studies showing belief-selective TPJ activation in explicit ToM reasoning tasks with older children and adults (Gweon et al., 2012; Hyde et al., 2015; Deen et al., 2015). Therefore, our results provide the first empirical evidence that systematic functional organization relevant for ToM is present in the temporal lobe within the first year of life and run contrary to predictions that such functional organization for ToM arises only later in childhood.

We also observed some evidence in both experiments that TPJ activity further distinguished scenarios involving FBs from a control condition (DP), in which the averted gaze of the person was matched with the FB condition, but the hiding locations were transparent, allowing the person direct perceptual access to the object's location (similar to the TB condition). The distinction between the FB and DP conditions provides additional evidence that the observed functional brain response reflects sensitivity to different mental states, or beliefs, rather than more general socially relevant variables such as direct versus averted gaze, perceptual access, or some other lower-level stimulus differences (Heyes, 2014). It is important to note that the FB/DP difference was only observed in the replication Experiment 2 with additional analyses not prespecified before data collection begun. Although we provide what we think is strong rationale and empirical validation for these additional analyses, we acknowledge this as a limitation in the evidence for replication of the differentiation between the FB and DP conditions in Experiment 2. Future work should build on these findings to better anticipate and 
A

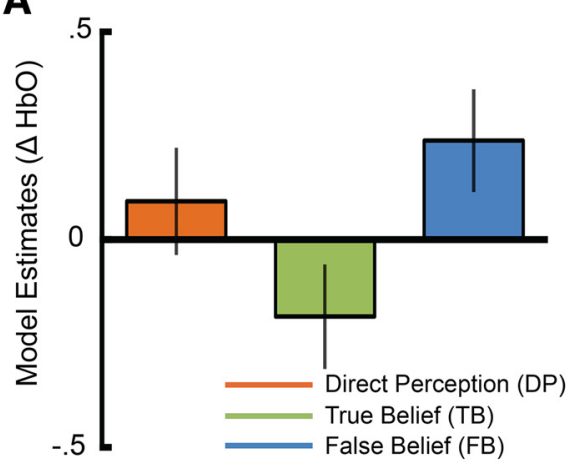

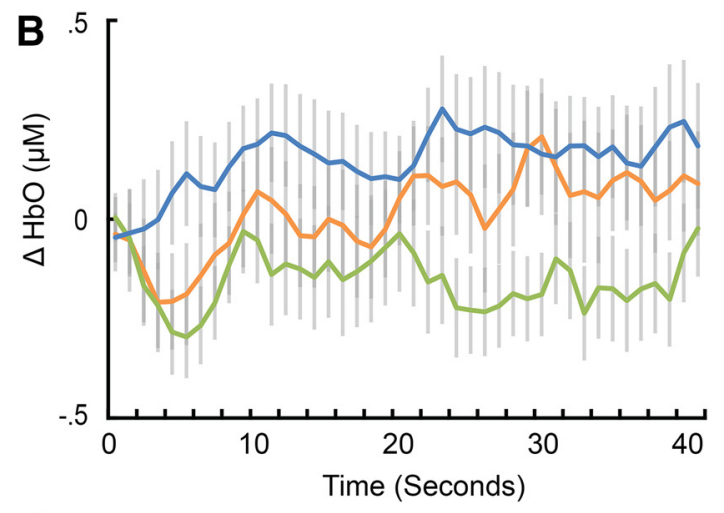

C

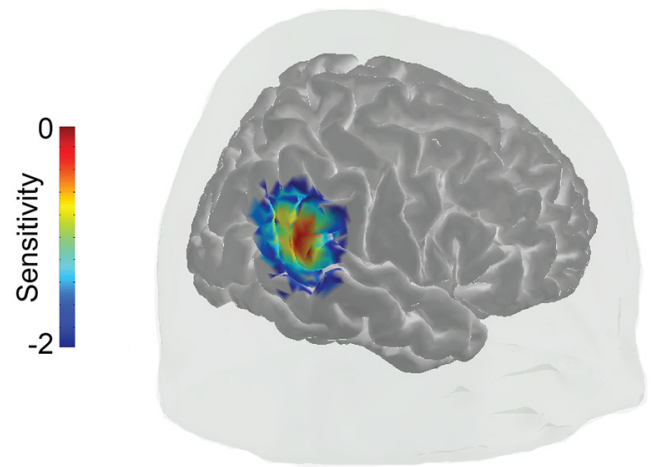

Figure 5. Experiment 2 results showing functional sensitivity to the beliefs of others in the TPJ. $A$, Estimates of least-squares means from the linear mixed model of belief condition on neural response of channel 3 between 22 and $26 \mathrm{~s}$. B. Time course of change in activity in channel 3 over the entire duration of the test videos. C, Cortical sensitivity estimates ( $\mathrm{mm}^{-1}$ ) for channel 3 displayed in $\log 10$ units (Materials and Methods for additional details). Error bars indicate $\pm 1 \mathrm{SE}$.
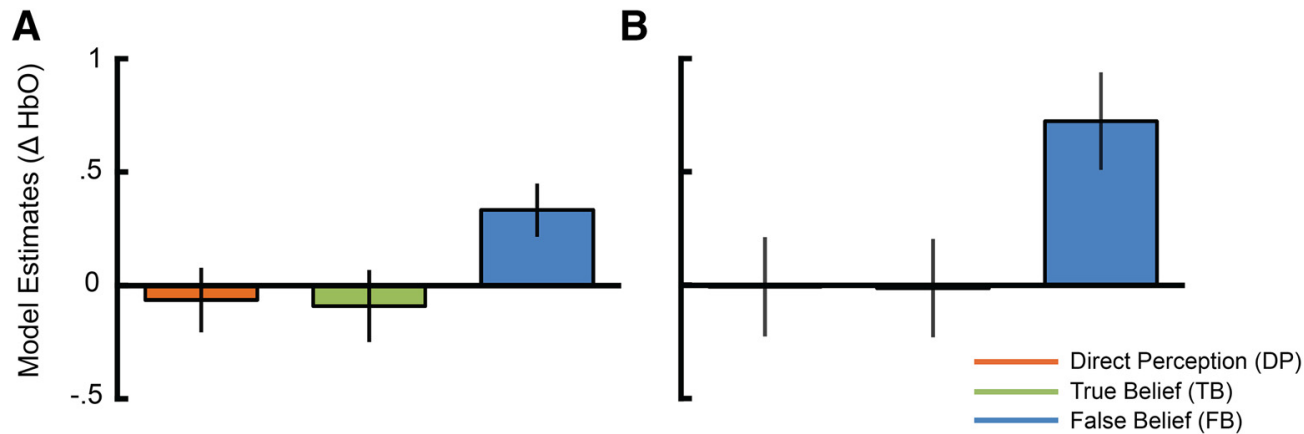

Figure 6. $\mathrm{fCOl}$ analysis showing sensitivity to the beliefs of others in the TPJ in both experiments. $A$, Experiment $2 \mathrm{fCOl}$ analysis. Bars represent least-squares means estimates from the linear mixed model of belief condition on neural response between 22 and $26 \mathrm{~s}$. $\boldsymbol{B}$, Experiment $1 \mathrm{fCO}$ analysis.

prespecify additional analyses to account for measurement variance before data collection begins. Even considering this limitation, though, we interpret the sum of the evidence across both experiments as supporting a functional response that is sensitive to beliefs, distinguishing FBs from both TB scenarios and DP control scenarios.

In contrast, we did not find robust evidence of right lateral prefrontal (Experiment 1) or left mPFC sensitivity (Experiment 2 ) to beliefs in infants. Although right lateral PFC is not thought to be specifically responsive to mental states in adults, regions of mPFC are considered part of the mature network of regions commonly attributed to ToM (Frith and Frith, 2012). There was a trend in the data suggesting that a portion of the MPFC may show a different functional response profile than the temporal lobe to our belief conditions during an earlier time window, with a greater response to direct versus averted gaze. However, this trend was found through entirely exploratory methods. Although the temporal portion of the ToM network may be functionally organized for tracking sociocognitive variables relevant to ToM from infancy, the mPFC may not or may be tuned to different sociocognitive variables. Further work is needed before any firm conclusions about the functional sensitivity of the infant mPFC in relation to ToM can be made.

In addition to illuminating the question of when basic functional organization for ToM arises, our results have implications for psychological theories of ToM. Whether infants and toddlers rely on the same mechanisms to track implicitly others' beliefs as older children and adults do to reason explicitly and answer questions about them remains a topic of intense debate and study (Sirois and Jackson, 2007; Apperly and Butterfill, 2009; Baillargeon et al., 2010; Perner and Roessler, 2012; Heyes, 2014; Scott and Baillargeon, 2017; Powell et al., 2017b; Dorrenberg et 
al., 2018). Some propose that foundational ToM mechanisms underlie both implicit belief tracking in infancy and explicit reasoning about beliefs in later childhood and adulthood (Leslie et al., 2004; Baillargeon et al., 2010; Carruthers, 2013). Others propose that implicit belief tracking in infancy draws on distinct mechanisms from those used for explicit belief reasoning later in life (Wellman et al., 2001; Apperly and Butterfill, 2009; Perner and Roessler, 2012; Heyes, 2014). The fundamental challenge in resolving this debate has been reconciling the evidence from distinct behavioral measures (implicit looking time/eye gaze vs explicit verbal or decision behavior) across the different age groups (infants vs older children and adults) to infer mechanism. Here, we took a novel approach to gain insight into the mechanisms underlying infant sociocognitive abilities, measuring the functional brain response to other's beliefs in infants and comparing it directly with what we know about the neural basis of ToM in adults. Our findings suggest that infants engage similar TPJ mechanisms to track beliefs as adults and older children do to reason explicitly about them. The particular pattern of neural sensitivity seen robustly across both experiments, distinguishing scenarios involving FBs from those involving TBs, mirrors a critical behavioral signature many agree is indicative of ToM (for reviews, see Apperly and Butterfill, 2009; Wellman et al., 2001). Therefore, both the functional response and anatomical localization of this response provides support for theories of a common foundational mechanism underlying ToM from infancy.

Although we provide evidence for a foundational mechanism for ToM from infancy, the nature and conceptual depth of infant ToM remains elusive. Based on the sum of available evidence, we favor an interpretation of the observed pattern of neural sensitivity as reflecting representation and differentiation of the beliefs of others. This strong interpretation is consistent with a growing body of evidence across many different sources that some capacity for FB understanding is already present in infancy (Scott and Baillargeon, 2017). Our data allow us to rule out some low-level interpretations of the differences observed between conditions, including low-level stimulus differences, associations, and novelty (Heyes, 2014; Dorrenberg et al., 2018). However, from our data alone, we cannot entirely rule out the possibility that the observed neural sensitivity reflects a more impoverished sociocognitive representation such as tracking of the accuracy of a person's perceptual knowledge with regard to the object. It is also important to note that we presented scenarios in which mental state representations were always likely to be engaged and tested whether the infant brain was sensitive to different mental states. This approach is qualitatively different from those that investigate whether the brain is selectively engaged for belief processing by contrasting stimuli likely to engage belief processing with stimuli not likely to engage belief processing (Deen et al., 2015). Future work should progress on both fronts to further understand the degree of brain organization and specialization for mental states early in life.

Regardless of interpretation, our findings have potential implications for understanding atypical development. For example, some developmental disorders such as autism show marked impairments in social cognitive processing, in particular ToM, which only become detectable by current methods in childhood (Baron-Cohen et al., 1985). The basic knowledge of typical developmental organization and ability to study the spontaneous engagement of the ToM network and associated brain correlates within the first year of life may allow earlier monitoring of sociocognitive function in those at risk for such disorders.

Even if one accepts a strong mentalistic interpretation of the data, though, this should not be taken as evidence that there is no further developmental change for ToM. From the behavioral work alone, it is clear that children increase in their ability to demonstrate explicit understanding of others' beliefs in different tasks and across different situations (Baillargeon et al., 2010). Furthermore, previous work has shown that the TPJ continues to increase in specialization for processing others' beliefs into late childhood and this brain development has been shown to be related to behavioral competency on explicit ToM tasks (Sabbagh et al., 2009; Saxe et al., 2009; Gweon et al., 2012; Bowman et al., 2015). Nevertheless, our results suggest that a central component of the mature ToM network, the TPJ, differentiates spontaneously between the belief states of others in infancy and that this sensitivity is present well before the capacity to explicitly demonstrate ToM develops. Therefore, our results support the notion of basic continuity in some critical brain and cognitive mechanisms for ToM from infancy.

\section{References}

Aasted CM, Yücel MA, Cooper RJ, Dubb J, Tsuzuki D, Becerra L, Petkov MP, Borsook D, Dan I, Boas DA (2015) Anatomical guidance for functional near-infrared spectroscopy: AtlasViewer tutorial. Neurophotonics 2:20801. CrossRef Medline

Apperly IA, Butterfill SA (2009) Do humans have two systems to track beliefs and belief-like states? Psychol Rev 116:953-970. CrossRef Medline

Aslin RN, Shukla M, Emberson LL (2015) Hemodynamic correlates of cognition in human infants. Annu Rev Psychol 66:349-379. CrossRef Medline

Baillargeon R, Scott RM, He Z (2010) False-belief understanding in infants. Trends Cogn Sci 14:110-118. CrossRef Medline

Baron-Cohen S, Leslie AM, Frith U (1985) Does the autistic child have a "theory of mind"? Cognition 21:37-46. CrossRef Medline

Barr DJ, Levy R, Scheepers C, Tily HJ (2013) Random effects structure in mixed-effects models: keep it maximal. J Mem Lang 68:255-278. CrossRef Medline

Bates D, Mächler M, Bolker BM, Walker SC (2015) Fitting linear mixedeffects models using lme4. J Stat Softw 67:1-48. CrossRef

Boas D, Culver J, Stott J, Dunn A (2002) Three dimensional monte carlo code for photon migration through complex heterogeneous media including the adult human head. Opt Express 10:159-170. CrossRef Medline

Bowman LC, Kovelman I, Hu X, Wellman HM (2015) Children's beliefand desire-reasoning in the temporoparietal junction: evidence for specialization from functional near-infrared spectroscopy. Front Hum Neurosci 9:560. CrossRef Medline

Carruthers P (2013) Mindreading in infancy. Mind Lang 28:141-172. CrossRef

Cooper RJ, Selb J, Gagnon L, Phillip D, Schytz HW, Iversen HK, Ashina M, Boas DA (2012) A systematic comparison of motion artifact correction techniques for functional near-infrared spectroscopy. Front Neurosci 6:147. CrossRef Medline

Custo A, Boas DA, Tsuzuki D, Dan I, Mesquita R, Fischl B, Grimson WE, Wells W 3rd (2010) Anatomical atlas-guided diffuse optical tomography of brain activation. Neuroimage 49:561-567. CrossRef Medline

Deen B, Koldewyn K, Kanwisher N, Saxe R (2015) Functional organization of social perception and cognition in the superior temporal sulcus. Cereb Cortex 25:4596-4609. CrossRef Medline

Dorrenberg S, Rakoczy H, Liszkowski U (2018) How (not) to measure infant theory of mind: testing the replicability and validity of four nonverbal measures. Cogn Dev. In press.

Edwards LA, Wagner JB, Simon CE, Hyde DC (2016) Functional brain organization for number processing in pre-verbal infants. Dev Sci 19:757769. CrossRef Medline

Fang Q (2010) Mesh-based monte carlo method using fast ray-tracing in plucker coordinates. Biomed Opt Express 1:165-175. CrossRef Medline

Frith CD, Frith U (2012) Mechanisms of social cognition. Annu Rev Psychol 63:287-313. CrossRef Medline

Gervain J, Mehler J, Werker JF, Nelson CA, Csibra G, Lloyd-Fox S, Shukla M, Aslin RN (2011) Near-infrared spectroscopy: a report from the McDonnell infant methodology consortium. Dev Cogn Neurosci 1:22-46. CrossRef Medline

Gweon H, Saxe R (2013) Developmental cognitive neuroscience of theory 
of mind. In: Neural circuit development and function in the brain (J Rubenstein \& P Rakic, eds), pp. 367-377. New York, NY: Elsevier.

Gweon H, Dodell-Feder D, Bedny M, Saxe R (2012) Theory of mind performance in children correlates with functional specialization of a brain region for thinking about thoughts. Child Dev 83:1853-1868. CrossRef Medline

Heyes C (2014) False belief in infancy: a fresh look. Dev Sci 17:647-659. CrossRef Medline

Huppert TJ, Diamond SG, Franceschini MA, Boas DA (2009) HomER: a review of time-series analysis methods for near-infrared spectroscopy of the brain. Appl Opt 48:D280-D298. Medline

Hyde DC, Boas DA, Blair C, Carey S (2010) Near-infrared spectroscopy shows right parietal specialization for number in pre-verbal infants. Neuroimage 53:647-652. CrossRef Medline

Hyde DC, Aparicio Betancourt M, Simon CE (2015) Human temporalparietal junction spontaneously tracks others' beliefs: a functional nearinfrared spectroscopy study. Hum Brain Mapp 36:4831-4846. CrossRef Medline

Koster-Hale J, Saxe R (2013) Theory of mind: a neural prediction problem. Neuron 79:836-848. CrossRef Medline

Kovács ÁM, Téglás E, Endress AD (2010) The social sense: susceptibility to others' beliefs in human infants and adults. Science 330:1830-1834. CrossRef Medline

Leslie AM, Friedman O, German TP (2004) Core mechanisms in "theory of mind.” Trends Cogn Sci 8:528-533. CrossRef Medline

Lloyd-Fox S, Blasi A, Elwell CE (2010) Illuminating the developing brain: the past, present and future of functional near infrared spectroscopy. Neurosci Biobehav Rev 34:269-284. CrossRef Medline

Lloyd-Fox S, Richards JE, Blasi A, Murphy DGM, Elwell CE, Johnson MH (2014) Coregistering functional near-infrared spectroscopy with underlying cortical areas in infants. Neurophotonics 1:25006. CrossRef

Luo Y, Baillargeon R (2007) Do 12.5-month-old infants consider what objects others can see when interpreting their actions? Cognition 105:489512. CrossRef Medline

Luo Y, Johnson SC (2009) Recognizing the role of perception in action at 6 months. Dev Sci 12:142-149. CrossRef Medline

Matsui M, Homae F, Tsuzuki D, Watanabe H, Katagiri M, Uda S, Nakashima M, Dan I, Taga G (2014) Referential framework for transcranial anatomical correspondence for fNIRS based on manually traced sulci and gyri of an infant brain. Neurosci Res 80:55-68. CrossRef Medline

Obrig H, Neufang M, Wenzel R, Kohl M, Steinbrink J, Einhäupl K, Villringer A (2000) Spontaneous low frequency oscillations of cerebral hemody- namics and metabolism in human adults. Neuroimage 12:623-639. CrossRef Medline

Onishi KH, Baillargeon R (2005) Do 15-month-old infants understand false beliefs? Science 308:255-258. CrossRef Medline

Perner J, Roessler J (2012) From infants' to children's appreciation of belief. Trends Cogn Sci 16:519-525. CrossRef Medline

Powell LJ, Deen B, Saxe R (2017a) Using individual functional channels of interest to study cortical development with fNIRS. Dev Sci. In press. CrossRef Medline

Powell LJ, Hobbs K, Bardis A, Carey S, Saxe R (2017b) Replications of implicit theory of mind tasks with varying representational demands. Cogn Dev. In press.

Sabbagh MA, Bowman LC, Evraire LE, Ito JM (2009) Neurodevelopmental correlates of theory of mind in preschool children. Child Dev 80:11471162. CrossRef Medline

Saxe RR, Whitfield-Gabrieli S, Scholz J, Pelphrey KA (2009) Brain regions for perceiving and reasoning about other people in school-aged children. Child Dev 80:1197-1209. CrossRef Medline

Scholkmann F, Spichtig S, Muehlemann T, Wolf M (2010) How to detect and reduce movement artifacts in near-infrared imaging using moving standard deviation and spline interpolation. Physiol Meas 31:649-662. CrossRef Medline

Scott RM, Baillargeon R (2017) Early false-belief understanding. Trends Cogn Sci 21:237-249. CrossRef Medline

Sirois S, Jackson I (2007) Social cognition in infancy: a critical review of research on higher order abilities. Eur J Dev Psychol 4:46-64. CrossRef

Southgate V, Vernetti A (2014) Belief-based action prediction in preverbal infants. Cognition 130:1-10. CrossRef Medline

Southgate V, Senju A, Csibra G (2007) Action anticipation through attribution of false belief by 2-year-olds. Psychol Sci 18:587-592. CrossRef Medline

Strangman G, Culver JP, Thompson JH, Boas DA (2002) A quantitative comparison of simultaneous BOLD fMRI and NIRS recordings during functional brain activation. Neuroimage 17:719-731. CrossRef Medline

Strangman G, Franceschini MA, Boas DA (2003) Factors affecting the accuracy of near-infrared spectroscopy concentration calculations for focal changes in oxygenation parameters. Neuroimage 18:865-879. CrossRef Medline

Wellman HM, Cross D, Watson J (2001) Meta-analysis of theory-of-mind development: the truth about false belief. Child Dev 72:655-684. CrossRef Medline 